\title{
Instagram: A niche for microlearning of undergraduate medical microbiology
}

\author{
I I Osaigbovo, ${ }^{1,2}$ MBBS, FMCPath; C F Iwegim, ${ }^{2}$ MBBS, MHPM \\ ${ }^{1}$ Department of Medical Microbiology, School of Medicine, College of Medical Sciences, University of Benin, Nigeria \\ ${ }^{2}$ Department of Medical Microbiology, University of Benin Teaching Hospital, Nigeria
}

Corresponding author: I I Osaigbovo (zephyreternal@yahoo.com)

\section{Context and setting}

In Nigerian medical schools, lecturing is the dominant mode of delivering education during the preclinical years. The School of Medicine, University of Benin (UNIBEN), Nigeria, a first-generation institution, provided the stage for the intervention described in this report.

\section{Why the idea was necessary}

The traditional lecture is fraught with pitfalls, such as cognitive overload and low engagement between students and faculty. ${ }^{[1]}$ These problems can potentially be tackled by social media. ${ }^{[2]}$ Scholars in Europe and the USA have described the innovative use of social media in medical education, but little documented evidence exists to suggest that African educators are up to date with this trend. We designed and implemented an intervention to complement face-to-face lectures.

\section{What was done}

The presence and activity of students on social networking sites during their pathology year was determined using a pre-intervention survey. Facebook and the photo-sharing application, Instagram, had the most users. Respondents accessed these sites predominantly via smart phones.

Content derived from the medical microbiology curriculum was posted on a dedicated Facebook page and Instagram account throughout the semester. Typical posts representing independent units of instruction consisted of an image accompanied by a concise descriptive text. A picture puzzle was also posted weekly. Students were provided with the account handles to voluntarily follow and engage on any or both social media platforms. An end-of-semester post-intervention survey was conducted.

\section{Results and impact}

There was a high level of satisfaction among participants; $91.6 \%$ agreed that the intervention enhanced learning. Specifically, posts allowed convenient, asynchronous access to content in manageable chunks (87.5\%), addressed gaps in knowledge (66.7\%) and afforded students opportunities to engage with their peers and facilitator (50\%). Participation and engagement were higher on Instagram than Facebook (70 v.14 followers; 2 students followed both). Furthermore, $85 \%$ of respondents reported deliberately using the platform as a mobile reference and $79.2 \%$ used it as a study tool during downtime, usually between lectures and while commuting. Accessing bite-sized content via mobile technology as and when required, whether for deliberate reference or opportunistic studying, has been described as 'microlearning. ${ }^{[3]}$ Coincidentally, these microlearning activities were reported exclusively by Instagram users.

Our take-home message from the intervention was twofold. First, the success of social media interventions is dependent on the students being positively motivated to use the selected platform. Our students possessed both Facebook and Instagram accounts, but had a greater affinity for the latter. Therefore, a thorough analysis of students' perceptions is vital before adopting any strategy. Secondly, the outcomes suggest that Instagram is more suitable for microlearning than Facebook. We attribute this to the succinct nature of the descriptive texts and the layout of the Instagram account, which allows images to function as bookmarks for posts of interest.

This intervention's highest contribution to scholarship in medical education lies in the new questions posed: what is the impact of microlearning as a pedagogical approach?; and is Instagram a more suitable vehicle than Facebook and other social media platforms for such an approach? Our future research will be directed at providing answers.

\section{Acknowledgements. None.}

Author contributions. IIO was responsible for the concept, implementation, acquisition and analysis of the data, and writing of the draft. CFI was responsible for the concept, revision of the draft and intellectual content.

Funding. None.

Conflicts of interest. None.

\footnotetext{
1. Frederick P. The lively lecture -8 variations. Coll Teach 1986;34(2):43-50. https://doi.org/10.1080/87567555.1 986.9926766

2. Orsini C, Evans P. Social media as a teaching strategy: Opportunities and barriers. Adv J Health Professions Educ 2015;1(1):44-46.

3. Hug T. Mobile learning as microlearning. Conceptual considerations towards enhancements of didactic thinking Int J Mobile Blend Learn 2010;2(4):47-57. https://doi.org/10.4018/jmbl.2010100104
}

Accepted 16 January 2018

Afr J Health Professions Educ 2018;10(2):75. DOI:10.7196/AJHPE.2018.v10i2.1057 